\title{
P. Alfons Friedrich buchreligion.de - oder: Der Versuch, auch heute dem religiösen Buch Präsenz zu geben
}

\section{Wenn etwas fehlt, fragt bald keiner mehr danach}

Der Buchhandel in Deutschland befindet sich - wie viele Wirtschaftszweige derzeit - in einer massiven Umbruchphase. Dies gilt insbesondere auch für den religiösen Buchmarkt, dessen Angebotsfläche in einem drastischen Ausmaß zurückgegangen ist. Mit dem Verschwinden z. B. der Herder-Buchhandlungen vom Markt begann ein Trend, der zunehmend Präsenzflächen für das religiöse Buch kostete. Heute fehlt selbst in vielen Bischofsstädten eine religiöse Buchhandlung. Von einer Grundversorgung mit christlicher Literatur für Christen oder einem überzeugenden Angebot für suchende Menschen kann keine Rede mehr sein. Durch das Entstehen einiger neuer Handelsflächen in Klöstern konnten zwar auch neue Anbieter hinzukommen, doch verändern diese wenigen positiven Lichtblicke keineswegs den Gesamttrend.

Es besteht also wesentlich ein fehlender Zugang zur religiösen Literatur, da zahlreiche konfessionelle Sortimenter vom Markt verschwunden sind bzw. das religiöse Sortiment in allgemeinen Buchhandlungen minimiert oder aufgegeben wurde. Angesichts dieser Entwicklung bedarf es verschiedener Versuche der Gegensteuerung, um zumindest eine Präsenz-Ermöglichung für das religiöse Buch zu schaffen.

Die zumindest gab es bis Anfang der Neunzigerjahre. Wer religiös/ theologische Bücher suchte, für den war „Das Katholische Schrifttum DKS“ ein Begriff. In diesem Verzeichnis - herausgegeben vom Verband katholischer Verleger und Buchhändler e. V. (VKB) - wurden alle lieferbaren religiös/theologischen Bücher nach einer speziellen Systematik aufgeführt.

Das evangelische Pendant nannte sich logischerweise „Das Evangelische Schrifttum - DES“ und wurde von der Vereinigung evangelischer Buchhändler und Verleger e. V. (VEB) in Stuttgart betreut. Sowohl dem Fachmann als auch dem Laien standen damit umfassende Nachschlagewerke für die Recherche im Bereich der christlichen Buchproduktion zur Verfügung. Die letzte Ausgabe des DKS erschien 1991, danach wurde diese Übersicht aus Kostengründen eingestellt. Für viele bedauerlich, hatte man sich doch im Laufe der Jahre daran gewöhnt, bei der Recherche zunächst auf das DKS zurück zu greifen. 
Fortgeführt wurde vorerst glücklicherweise „Theologie und Religion aktuell", eine zwei Mal jährlich erscheinende Übersicht über Neuerscheinungen mit religiös/theologischem Inhalt, ebenfalls mit einer speziellen Verschlagwortung. Herausgegeben vom VKB/Kath. Medienverband, erschien es im Herder Verlag Freiburg und wurde inhaltlich von Horst Rummel betreut.

Doch sowohl Buchhändler/-innen als auch deren Kundinnen und Kunden im religiösen Segment vermissten nun eine fachliche Übersicht aller lieferbaren Titel. Dies war auch den Verantwortlichen im VKB schnell klar, und so gab es erste Überlegungen für ein Nachfolgeprodukt. In dieser Zeit begann der Personalcomputer Einzug zu halten in die Wohnund Arbeitszimmer der deutschen Haushalte und die Anbindung dieser PCs an das Internet nahm zu. Wie also konnte ein möglicher Nachfolger des DKS aussehen?

\section{Neue Medien - neue Chancen?}

$\mathrm{Zu}$ jener Zeit erschien erstmalig das "Verzeichnis lieferbarer Bücher" (VLB) auf einer $C D$, dieses Medium begann sich als Datenträger für größere Datenbestände durchzusetzen. Warum also nicht die lieferbaren religiösen Titel auch über eine CD erschließbar machen? Auch war auf einer CD genug Platz, nicht nur für die katholischen Titel, sondern ebenfalls für den Datenbestand des DES, das inzwischen das gleiche Schicksal wie das DKS erleiden musste. Die technische Entwicklung im PCBereich boomte in diesen Jahren und das Internet wurde als neuer Absatzkanal von vielen Unternehmen - vom Buchhandel zunächst noch zaghaft - entdeckt. Damit kam eine weitere Aufgabenstellung hinzu: Wenn schon eine Recherchemöglichkeit für alle christlichen Titel eingerichtet wird, sollte man dann nicht direkt auch die Bestellmöglichkeit mit anbieten? Natürlich war mittlerweile auch das VLB online, doch es bot eben nicht die Vorzüge des DKS und DES.

Zwei generelle Fragen stellten sich bei den Überlegungen zur Realisierung eines solchen Projektes. Wie wird es finanziert und wie bekommt man den notwendigen Datenbestand? Ganz klar war, dass eine Internetdatenbank nicht alleine aus Mitteln des VKB finanziert werden konnte. Der Vorstand des VKB wandte sich an die Deutsche Bischofskonferenz und bat um Unterstützung für „reli-book.de“, so der erste Arbeitstitel.

Auf der einen Seite mussten viele christliche Buchhandlungen schlie ßen, auf der anderen Seite hatte das Internet als Rechercheinstrument und Bestellweg kontinuierlich an Bedeutung gewonnen. Die Online umsätze des Buch- und Medienhandels stiegen, trotz schwieriger wer- 
dender wirtschaftlicher Rahmenbedingungen. Auch die Deutschen Bischöfe erkannten diesen Trend und sagten dem Projekt ihre - auch finanzielle - Unterstützung zu. Bei der Umsetzung des Projektes stand die Medien-Dienstleistungs Gesellschaft (MDG) beratend zur Seite.

„reli-book.de“ sollte also umfassende Recherche- und Bestellmöglichkeiten für Fachleute und Laien bieten. Umfassend, sprich alle lieferbaren Titel bereitzustellen, das war seinerzeit der Anspruch des DKS, der nun auf das neue Medium übertragen werden sollte. Was also lag näher, als auf den Anbieter zuzugehen, der den kompletten Datenbestand der lieferbaren Titel verwaltet, nämlich die VLB-Redaktion. Aus dem VKB war auf dem Wege der Verschmelzung mit der Arbeitsgemeinschaft Katholische Presse (AKP) mittlerweile der Katholische Medienverband (KM) geworden.

Waren die ersten Überlegungen noch davon ausgegangen, nur die katholischen Titel in dieser Datenbank zu erfassen, entschieden die jeweiligen Vorstände in den beiden Verbänden, dass nur ein gemeinsames Vorgehen von KM und VEB, mit einem gemeinsamen ökumenischen Auftritt, sinnvoll ist. Man kam schnell überein, die jeweiligen Konzeptionen für einen Internetauftritt zusammenzuführen und eine gemeinsame Plattform für Fachleute und Laien zu bieten. Und auch hier war das VLB mit seinem umfassenden Datenbestand wieder der bevorzugte Partner.

\section{buchreligion.de - Bücher und mehr}

In Zusammenarbeit mit der Marketing- und Verlagsgesellschaft des Buchhandels (MVB) sind nun seit Juli 2003 ca. 55000 religiöse Titel über das Internetportal www.buchreligion.de recherchier- und über ein Netzwerk stationärer Buchhandlungen bestellbar.

Dies ist aus dem Bestand von über einer Million lieferbaren deutschsprachigen Büchern die Menge, die von der VLB-Redaktion als Titel mit religiös/theologischen Inhalten gekennzeichnet worden sind. Somit sind alle lieferbaren christlichen Titel im Prinzip rund um die Uhr und weltweit recherchier- und bestellbar. Zahlreiche Titel sind nicht nur mit den reinen bibliografischen Angaben zu finden, sondern bieten darüber hinaus Coverabbildungen und zusätzliche Texte mit weiter gehenden Informationen über das ausgewählte Buch.

buchreligion.de bietet auch Zugang zu CDs, DVDs und MCs, die über den Sortimentsbuchhandel vertrieben werden. In einer für 2004 geplanten Erweiterung des Portals sollen auch christliche Zeitschriften und Zeitungen zugänglich gemacht werden und in einer nächsten Ausbaustufe der Bereich der „non-books“, also Devotionalien, Klosterprodukte u. ä. 
Mit der Bereitstellung des Portals und Anbindung an das VLB konnte über das ursprüngliche Ziel hinaus, nämlich einen „Nachfolger“ für DKS und DES zu schaffen, auch noch eine Bezugsmöglichkeit geschaffen werden, die die Erhältlichkeit christlicher Buch- und Medientitel ganz allgemein erleichtert und verbessert.

Was ist aber das Besondere an buchreligion.de, wo liegen die Vorzüge gegenüber dem VLB?

Für buchreligion.de wurde von Fachleuten aus den beiden christlichen Verbänden eine besondere Schlagwortsystematik entwickelt, die über die bisher bestehende und im VLB angewandte Systematik hinausgeht. "Christliches Leben“, „Kinder und Jugendliche“, „Kirchliche Praxis“ und "Wissenschaftliche Theologie“ lauten die Hauptbegriffe, innerhalb derer mit weiteren Schlagwörtern gezielt nach Titeln gesucht werden kann. So bietet z. B. das Schlagwort „Kirchliche Praxis“ in der Rubrik „Gemeindearbeit/-katechese/-pädagogik“ die Untergruppe „Seniorenarbeit“ an, in der alle lieferbaren Titel zu diesem Thema mit christlich-religiösem Bezug gefunden werden können. Rund 100 Schlagwörter weist die Systematik auf, der Schlagwort-Index besteht aus rund 900 Begriffen.

Selbstverständlich kann auch über eine Suchmaske ganz gezielt nach einer/m bestimmten Autor/-in oder einem besonderen Titel gesucht werden. Einschränken lässt sich die Recherche auch nach Erscheinungsjahr und Ladenpreis, sodass über diese zahlreichen Selektionskriterien eine hohe Treffergenauigkeit erzielt werden kann. Weiterer Vorzug des Portals buchreligion.de ist die Möglichkeit - über die eingeschränkte Suche eben jener rund 55000 Titel hinaus - im gesamten Datenbestand des VLB zu recherchieren und $z u$ bestellen.

Die ausgewählten Titel werden in einen Warenkorb gelegt und können über eine der am Shopsystem angeschlossenen Mitgliedsbuchhandlungen des KM oder der VEB bestellt werden.

\section{Eine besondere Chance für das religiöse Sortiment}

Für die am Shopsystem teilnehmenden Buchhandlungen (und auch Verlage) bieten sich verschiedene Möglichkeiten. Alle teilnehmenden Buchhandlungen erscheinen als mögliches Bestellziel in einer nach Postleitzahlen sortierten Liste. Darüber hinaus kann das Portal in eine bestehende Homepage integriert werden und die Buchhandlung das Portal buchreligion.de ganz gezielt für das eigene Unternehmen als Marketinginstrument nutzen.

Das Portal verzeichnet zurzeit ca. 6000 Seitenzugriffe pro Monat mit steigender Tendenz. 
Doch nicht nur für Buchhandlungen und -verlage ist buchreligion.de ein weiteres Standbein. Auch christliche (Zeitschriften- und Zeitungs-) Verlage können sich am Shopsystem beteiligen, sofern die Unternehmen Mitglied im KM oder der VEB sind. Damit erschließt sich diesen Firmen, die bisher vielleicht über einen eigenen Vertriebskanal ein eingeschränktes (verlagseigenes) Angebot bereit gestellt haben, die ganze Bandbreite christlicher Bücher und Medien, darüber hinaus sogar der komplette Bestand der lieferbaren Bücher.

Die zahlreichen Zusatzinformationen, die mittlerweile bei vielen Titeln u. a. auch zur Person der Autorin/des Autors hinterlegt sind, geben auch Redakteurinnen und Redakteuren für ihre tägliche Arbeit eine wertvolle Hilfestellung bei der Recherche an die Hand. Rezensionen oder Buchvorstellungen können, sofern nicht bereits mit einer örtlichen Buchhandlung kooperiert wird oder ein eigener Vertriebskanal vorhanden ist, den Hinweis auf buchreligion.de enthalten und so den Service für die Leser erhöhen.

Verlagen wird die Möglichkeit geboten, ausgewählte Titel auf der Portal- und den Folgeseiten für einen begrenzten Zeitraum ausführlich vorzustellen. Über einen Link kann der Titel direkt in den Warenkorb gelegt werden.

Darüber hinaus kann das Portal auch noch für weitere Anbieter im christlichen Bereich von Interesse sein. Mittlerweile existieren zahlreiche kirchliche (Weiter-)Bildungshäuser und -einrichtungen, die als Ergänzung zu ihren Angeboten auf buchreligion.de verlinken und in ihren Veranstaltungen auf das Portal hinweisen könnten. Noch effektiver erscheint die Kooperation mit einer in der Nähe gelegenen Buchhandlung.

\section{Was wird kommen?}

In einer Zeit, in der die Kirchenaustritte nicht weniger werden, stellt sich für viele dennoch immer wieder die Frage nach dem Sinn, zeigt sich ein Bedürfnis nach Einkehr und Selbstfindung.

Auch aus diesem Grund verzeichnen viele der Bildungshäuser und Klöster, die Einkehrtage anbieten, immer stärkeren Zulauf. Und noch immer ist das Buch auf dem Weg zu Beantwortung der Fragen „Wo komme ich her, wo gehe ich hin?" ein wertvoller Ratgeber.

Wenn es gelingt, alle diejenigen Personen, Unternehmen und Institutionen, die mit ihrem jeweiligen Angebot Unterstützung auf diesem Weg leisten können, in einem Netzwerk zusammen zu fassen, profitieren letztendlich alle Beteiligten davon.

Hierzu möchte das Portal www.buchreligion.de seinen Beitrag leisten. 